\title{
Evaluación de riesgos y modelación de soluciones técnicas para filtraciones en presas de tierra
}

\section{(Risk assessment and modeling of technical solutions for filtrations earth dams)}

\author{
Michael Álvarez González ${ }^{1,2}$, Lamberto Álvarez Gil², Romel Vázquez Rodríguez ${ }^{2,3}$
}

\begin{abstract}
Resumen:
En el presente trabajo se hace la evaluación y el análisis de tres tipos de geometría de filtros para evaluar el riesgo de las filtraciones en el Embalse Zaza, a partir de las características históricas de las fluctuaciones de niveles de agua dentro de la presa de tierra. El trabajo se basa en la experiencia de un equipo multidisciplinario e investigación previa con modelos bidimensionales usando el Método de los Elementos Finitos en la solución de problemas básicos en la ingeniería. También se evalúan los resultados de la colocación del nuevo sistema de filtros con un indizado geoespacial (bajo los criterios de: amenaza, vulnerabilidad y riesgo) con datos espaciales de visualización en un Sistema de Información Geográfica para la generación de mapas temáticos que representan cuanto varía el nivel del agua en el interior de la cortina a partir de los diferentes tipos de filtros evaluados.
\end{abstract}

Palabras clave: presa de tierra; método de los elementos finitos; análisis de riesgo; sistema de información geográfica; índice geoespacial.

\begin{abstract}
:
The paper presents the evaluation and the analysis of three types of filter geometry to evaluate the filtration risk in Zaza Reservoir, taking as starting point the historic characteristics of the fluctuation of water levels inside the earth dam. The work is based on the experience of a multidisciplinary team and previous research with bidimensional models, using the Finite Elements Method for the solution of basic engineering problems. Also, the results of the installation of a new filter system with a geospatial index are evaluated (under criteria of threats, vulnerability, and risk) with spatial visualization data in a Geographical Information System for thematic maps generation, that represent how much the water level varies inside the dam according to the different filters evaluated.
\end{abstract}

Keywords: earth dam; finite element method; risk analysis; geographic information system; geospatial index.

\section{Introducción}

El proceso de la evaluación de riesgos y simulación de soluciones técnicas como una disciplina de rigor en el campo de las soluciones ingenieriles, utilizadas como herramientas previas al proceso de restauración o reparación de obras hidráulicas es una línea de desarrollo actual por la comunidad científica mundial. El desarrollo de la tecnología y de la informática aplicada a diversos campos de la ingeniería, hace posible que la evaluación de riesgos y simulación de una solución técnica a problemas básicos de la ingeniería sean realizados previamente en laboratorios virtuales. A partir de la

\footnotetext{
${ }^{1}$ Empresa de Investigaciones y Proyectos Hidráulicos de Villa Clara (IPH VC), Santa Clara, Villa Clara - Cuba (michael@vc.hidro.cu).

2 Universidad Central «Marta Abreu» de Las Villas, Santa Clara, Villa Clara - Cuba (lambertoag@uclv.edu.cu).

3 Universidad Metropolitana del Ecuador (UMET), Quito - Ecuador (vromel@umet.edu.ec).
} 
caracterización de los materiales utilizados en el proceso constructivo de la obra previos se aporta un considerable ahorro de recursos técnicos y humanos en función de una adecuación medioambiental y tecnológica al proceso constructivo en sí mismo.

Para el caso específico de las presas de tierra, la acción de empuje del agua embalsada provoca movimientos significativos en la cortina del embalse. La variabilidad de los niveles de agua dentro del medio permeable que conforma la Línea de Corriente Superior (LCS), es otro de estos elementos técnicos que se estudian en detalles para definir un área o volumen de material que está completamente saturado. Definir la zona de principal interés ante posibles filtraciones a causa de la variabilidad conductiva del medio poroso que se manifiesta en el talud de aguas abajo implica a su vez la necesidad de evaluar la estabilidad y seguridad de la obra (M. Álvarez, L. Álvarez, y Vázquez, 2017).

En el caso de un proceso de diagnóstico y evaluación de las patologías se identifican los estudios presentados por Álvarez (1998) en varios momentos. En el trabajo se desarrolla una solución basada en el análisis de la estabilidad del talud en cortina de presas de tierra aplicando la teoría de seguridad. Esta teoría se consolida posteriormente en una lista de chequeo con las modelaciones físico-numéricas correspondientes para la creación de catálogos de patologías que evalúan las características e influencias en el proceso de seguridad de explotación del embalse objeto de estudio.

Durante el proceso de concepción tecnológica de un embalse se identifican los estudios presentados por (Sherald y Woodward, 1963) fomentándose bases de gran valor para futuras investigaciones de diseño de presas de tierra contenidas en las experiencias de varios autores (L. Álvarez, 1998; Armas, 1990, 2002; Armas, Echemendía, y Garcías, 1994; Armas y Horta, 1987; Botello, 2006; MICONS, 2005a, 2005b; Popnicolov, 1974). Estos, aportaron criterios actualizados al tema mediante el empleo de formulaciones empíricas, clásicas y novedosas soluciones en su momento.

Los estudios presentados por Bitner (2016) proponen interesantes métodos de integración y análisis de la información cartográfica por medio de los Sistemas de Información Geográfica.Estos resultados se pueden compatibilizar con las experiencias alcanzadas por Kasireddy et al. (2015), para gestionar una cartografía de riesgo a partir de los parámetros de diseño y monitoreo del embalse, elementos estos que sustentan bases teóricas en la presente investigación, así como otros resultados similares publicados por varios autores (Aydemir, A. y Güven, A., 2017; Cerón López, G. M., 2017; Daniszewska, E., 2017; Li, Y. y Wang, Y., 2017; Vázquez, R. et al., 2015).

Además, en correspondencia con las constantes variaciones climáticas y la ocurrencia de eventos extremos con mayor frecuencia, se hace necesario el estudio detallado de diversas patologías en función del proceso de las fluctuaciones de los niveles de agua en los embalses y las particularidades de cada uno de estos ante el proceso de posibles grietas interiores que conduzcan a un posterior fallo estructural de la cortina de la presa de tierra.Es por ello que evaluar soluciones técnico-ingenieriles a problemas básicos de la ingeniería desde la etapa de diseño, constituye un punto de partida necesario a desarrollar en el sector empresarial cubano, a fin del proceso de integración académica con sectores de vanguardia en ramas claves de la economía nacional.

La presente investigación se desarrolla en el campo de la ingeniería hidráulica utilizando herramientas de la modelación físico-numérica, para abordar como objeto de estudio la evaluación de proyectos en función de riesgos identificados a consecuencia de las patologías de filtraciones existentes en presas de tierra. Se asume como interrogante científica para el presente trabajo: ¿Cómo realizar una adecuada evaluación técnica de una solución ingenieril para la disminución de los riesgos por surgencia de la LCS en el cuerpo de la cortina de una presa de tierra?, asociado al caso de estudio: Embalse Zaza.

Además, se asume la siguiente hipótesis: si se realiza una adecuada evaluación técnica de la solución propuesta para la disminución de los riesgos asociados al proceso de surgencia de la LCS en el cuerpo de la cortina de presa de tierra, entonces será posible simular la efectividad técnica del proyecto en condiciones de laboratorios virtuales 
previo al proceso constructivo, y se reducirá el riesgo de falla del diseño en función de la solución al problema objeto de estudio en la presente investigación.

Por objetivo general se propone:

1. Evaluar riesgos y simular soluciones ingenieriles para redireccionar el flujo de agua en una pared en suelo al pie del talud de una presa de tierra.

Con los siguientes objetivos específicos:

1. Modelación físico-numérica empleando el Método de Elementos Finitos (MEF) de las filtraciones con el diseño o propuesta de solución técnica al problema de surgencia del nivel de agua en la cortina de la presa de tierra para determinar la LCS teórica antes y después de la propuesta técnica de solución.

2. Evaluación técnica de la solución ingenieril para la disminución de los riesgos por surgencia de la LCS en el cuerpo de una cortina de presa de tierra por medio de criterios de Amenaza Vulnerabilidad y Riesgo.

\section{Metodología}

El estudio fue desarrollado en el embalse Zaza, ubicado en la provincia de Santi Spíritus al sursureste de la ciudad de Santi Spíritus, Cuba (observar Figura 1); con los principales objetos de obras que existen en la zona de estudio a considerar como vulnerabilidades. Para la definición de la geometría que fue utilizada, se definió una sección central desplazada unos 3 metros aproximadamente a la izquierda de la galería, acorde con el sistema de referencia local del embalse. Dada la similitud predominante en la conformación de los taludes en la zona de influencia de las anomalías que fueron objeto de estudios en investigaciones previas publicadas por el autor (M. Álvarez, L. Álvarez, y Martínez, 2015a, 2015b), se decidió utilizar la misma información de base para darle continuidad a la investigación.

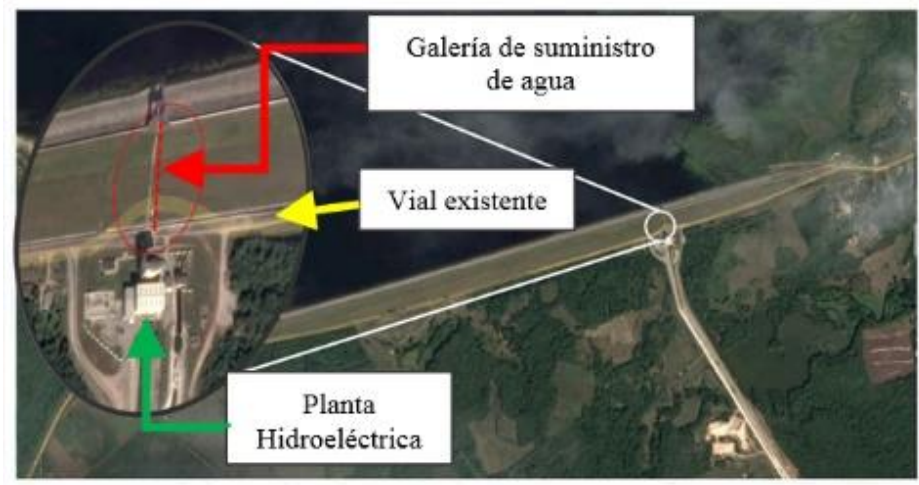

Figura 1. Área de estudio.

Para lograr un correcto y eficiente proceso de modelación se optó por el modelo bidimensional de los estados tenso-deformacionales en similares condiciones de diferentes análisis del comportamiento de los materiales (lineal-elástico, elasto-plástico e hiperbólico). Los resultados obtenidos por medio de los métodos de elementos finitos representándose presentaron en el pos-procesador gráfico para la visualización de datos para mantener una compatibilidad básica de la información. Al obtener la geometría de la sección actual deformada, se procedió a estimar la LCS teórica antes y después de la reparación, así como la colocación de los sistemas de filtro para tres tipos de geometrías y dimensiones diferentes en la sección trasversal del dren a construir.

Se analizaron la curva de filtración y las redes de flujo dentro de la cortina, para obtener el comportamiento de las mismas y estimar el gasto de agua aproximado que fluye por el interior de la estructura. El comportamiento del material empleado en el modelo fue asumido bajo condiciones de semisaturado y saturado. Utilizando los niveles 
del agua medidos en el interior de las calas realizadas a la cortina como patrón de calibración física al cálculo de las filtraciones teóricas en la maqueta virtual.

Fue chequeada la estabilidad de los taludes en la estructura, principalmente en el talud aguas abajo, por diferentes métodos: Fellenius, Bishop y Janbu. Esto permite determinar cuánto mejora o se deteriora el Factor de Seguridad del talud de cortina de acuerdo con las diferentes superficies de falla estimadas para la posición de la LCS antes y después de la restitución de la red de filtros de drenaje. Este proceso permitió determinar la estabilidad de las paredes en suelo de las tres geometrías al realizar el proceso de excavación en las variantes de geometrías consideradas para esta investigación ante la presencia de la presión de poros en el suelo y el posible fallo por el cambio del estado tensional del suelo.

Al disponer de todos los datos previos derivados de los cálculos por MEF se realizó un proceso de asignación de valores a los puntos de control de las calas según sus coordenadas en el terreno, creando una base de datos geo-espacial. Así, el problema de realizar un análisis puntual con MEF es descartado con un proceso de interpolación con elementos de la geo-estadística por medio del método Kriging Universal al visualizar diferentes escenarios en el tiempo. La respuesta resultante fue sometida a técnicas de geo-procesamiento de la información por lógica difusa para delimitar la respuesta de vulnerabilidad más probable por superposición geométrica de la interpolación de diferentes valores de niveles de agua en el tiempo en la base de datos creada.

El resultado fue representado en mapas temáticos para visualizar la variación espacial en toda la estructura de la LCS.

Para el control y lectura de las mediciones del nivel freático en el interior de la cortina del embalse se hicieron una total de 22 calas en diferentes posiciones a lo largo de la zona de estudio. Las características típicas y finalidad de las mismas son señaladas en las figuras 2 y 3). Como elemento de control fueron combinados con los datos provenientes de los piezómetros activos en la zona de interés válido de la investigación y señalado en color rojo la zona donde se pretende evaluar la solución técnica.

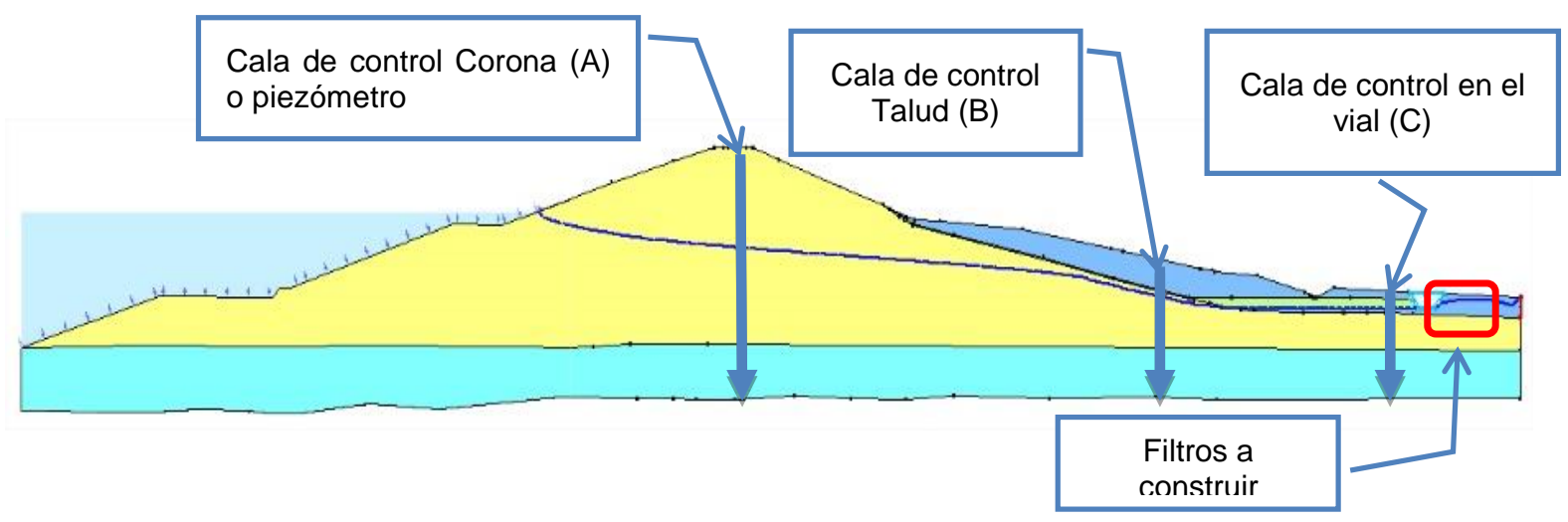

Figura 2. Características de las calas de control realizadas a la cortina del embalse. 


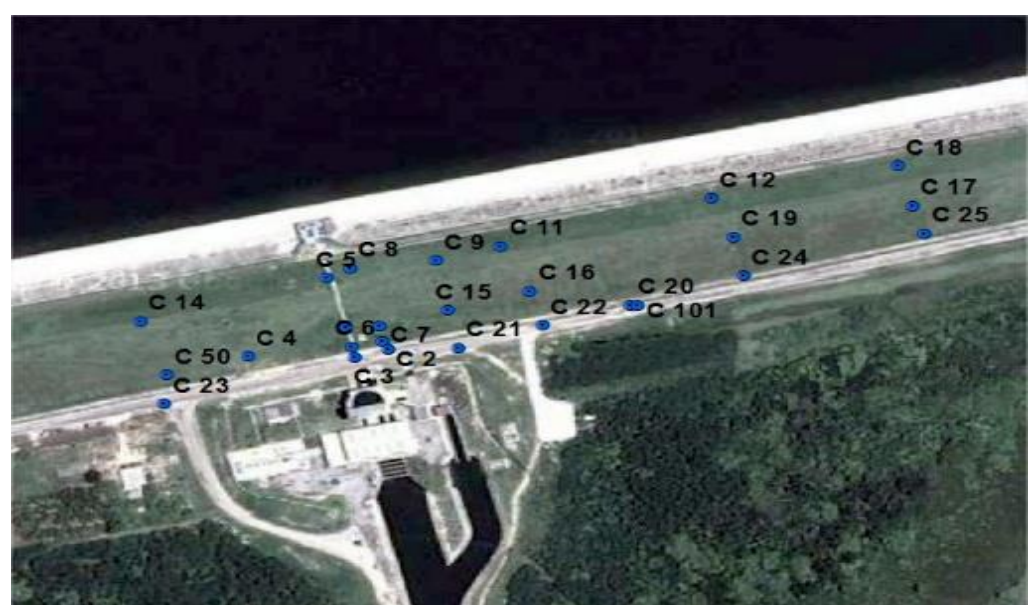

Figura 3. Distribución de las calas de control en la cortina del embalse.

Además, se dispuso de los registros históricos de los niveles de agua del embalse y de los valores correspondientes en los piezómetros y vertedores totalizadores para poder establecer un análisis correlacional histórico (observar Figura 4).

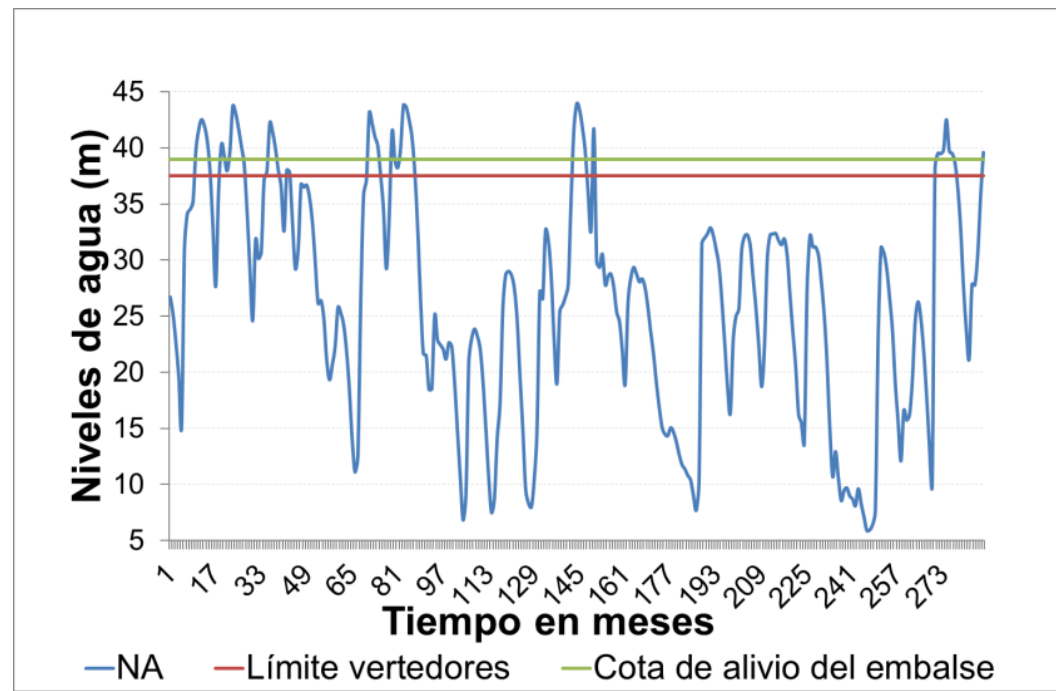
embalse

Figura 4. Representación gráfica de los niveles de agua históricos registrados en el

Las geometrías de las tres secciones transversales que fueron objeto de estudio fueron de forma trapezoidal, triangular y circular (obsérvese la Figura5) con variaciones en sus dimensiones y formas geométricas para valorar la sensibilidad de la respuesta de los modelos utilizados.



Figura 5. Secciones transversales modeladas 


\section{Resultados}

Los resultados de los niveles de agua alcanzados en el cálculo de la posición teórica de la LCS en el cuerpo de la cortina, fueron basados en las formulaciones del MEF y en el Método de Cálculo de Casagrande. El comportamiento probable de la curva de filtraciones (obsérvese la Figura 6) muestra una diferencia en cota no menor de $0.1 \mathrm{~m}$ en los diferentes puntos de control representados en la Figura 6 y la Tabla 1. Los datos reales medidos en las calas de control fueron comparados con los gastos calculados para la sección de estudio. Este análisis permitió comparar cuan efectiva es una solución geométrica del filtro modelada respecto a las demás.

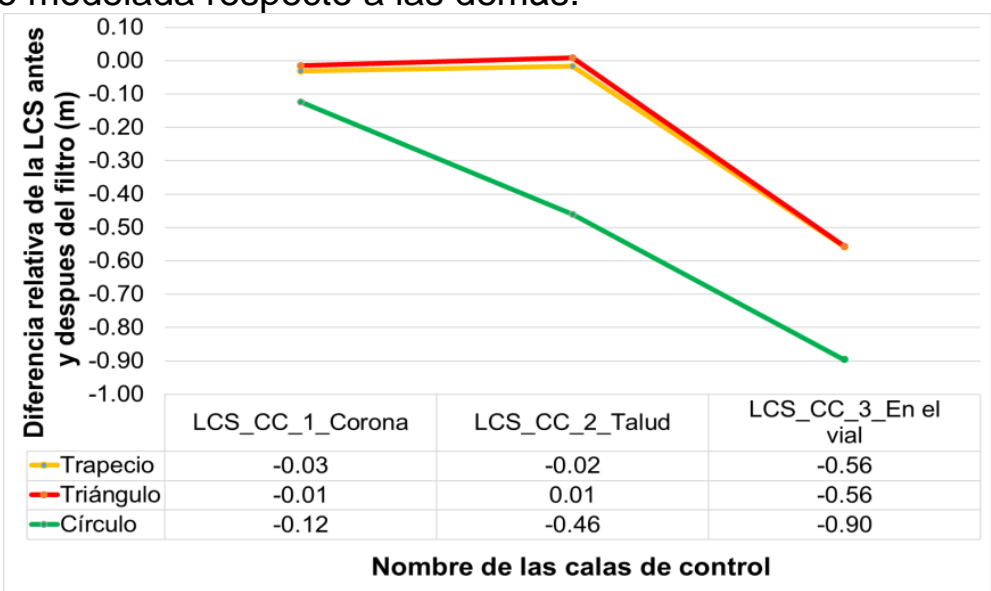

Figura 6. Diferencias de la LCS en las diferentes calas de control una vez diseñado el filtro.

Tabla 1. Cálculo de los gastos de filtraciones antes y después de haber modelado la solución técnica.

\begin{tabular}{|c|c|c|c|}
\hline \multirow[b]{2}{*}{$\begin{array}{c}\text { Tipo de } \\
\text { sección transversal }\end{array}$} & \multicolumn{3}{|c|}{ Gastos en las secciones $\left(\mathrm{m}^{3} / \mathrm{s}\right)$} \\
\hline & $\begin{array}{l}\text { Antes del } \\
\text { filtro }\end{array}$ & $\begin{array}{l}\text { Después del } \\
\text { filtro }\end{array}$ & Diferencia \\
\hline Trapecio & $2.81 \mathrm{E}-06$ & $2.49 \mathrm{E}-07$ & $2.56 \mathrm{E}-06$ \\
\hline Triángulo & $2.80 \mathrm{E}-06$ & $2.64 \mathrm{E}-07$ & $2.53 \mathrm{E}-06$ \\
\hline Círculo & $2.85 \mathrm{E}-06$ & $2.17 \mathrm{E}-07$ & $2.64 \mathrm{E}-06$ \\
\hline
\end{tabular}

Luego de haber realizado una evaluación del comportamiento de los filtros y haber modelado la reducción en cota que presenta la LCS en el interior de la cortina del embalse (observar figuras 7 y 8 ) se realizó una evaluación de control donde se compararon los valores de riesgo que fueron previamente identificados en años anteriores con los nuevos evaluados en un SIG.

Basado en la comparación mostrada en la Figura 6, se identificó una reducción en la cota de los niveles de agua en el interior de la estructura para todos los casos (o del nivel freático en los piezómetros y/o calas) y por consecuencia, los valores que se identificaron como un nivel de amenaza que sobrepasan los límites superiores de los niveles de agua pronosticados, son numéricamente cercanos a los valores obtenidos por el cálculo de la posición de la LCS por el Método de los Elementos Finitos acorde con el rango promedio de los valores iniciales del modelo de pronóstico LC_P11 (junio/agosto/septiembre) según los parámetros condicionantes de cada modelo evaluado en el tiempo (mes), luego de haber sido colocado el filtro (obsérvese la Figura9). 


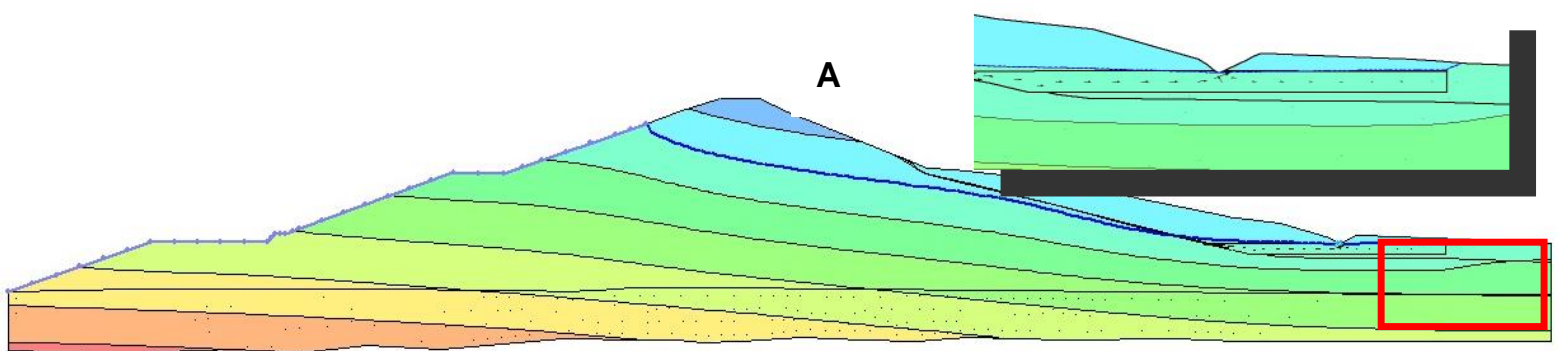

B

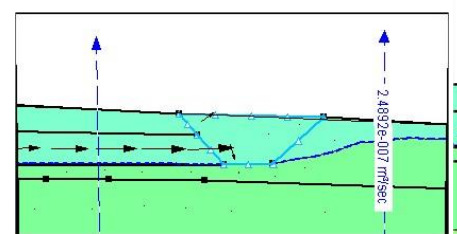

Filtro tipo prisma

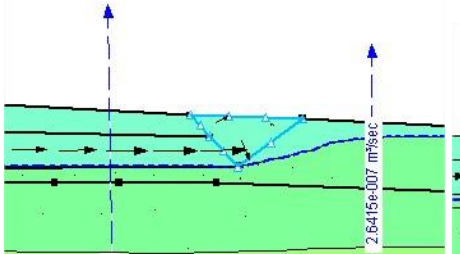

Filtro triangular

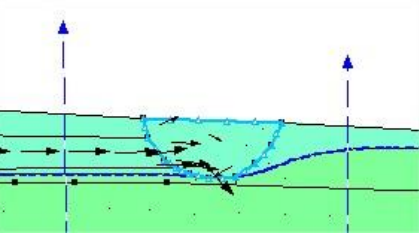

Filtro circular filtros(B).

Figura 7. Resultados calculados de la LCS sin filtro (A) y con las diferentes geometrías de

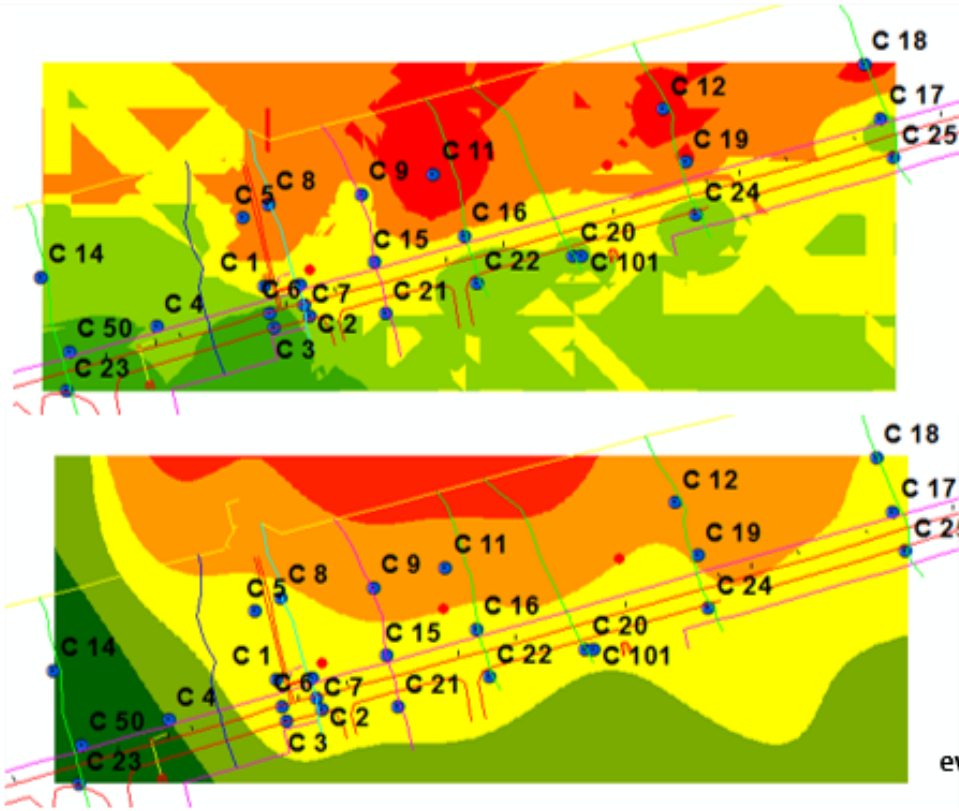

Leyenda (unidades en metros) $24.27-25.83$

$\square 25.84-26.38$

$\square 26.381-26.88$

$\square 26.88-27.38$

$27.38-29.58$

Leyenda (unidades en metros)

$24.19-24.96$

$\square 24.96-25.51$

$\square 25.51-25.94$

$\square 25.94-26.43$

$26.43-27.31$

Escala de colores acorde a la evaluación de los posibles escenarios.

\section{Riesgo}

Vulnerabilidad

\section{Amenaza}

Figura8. Distribuciones espaciales indizadas del valor más probable para la LCS antes del filtro $(A)$ y después del filtro $(B)$. 


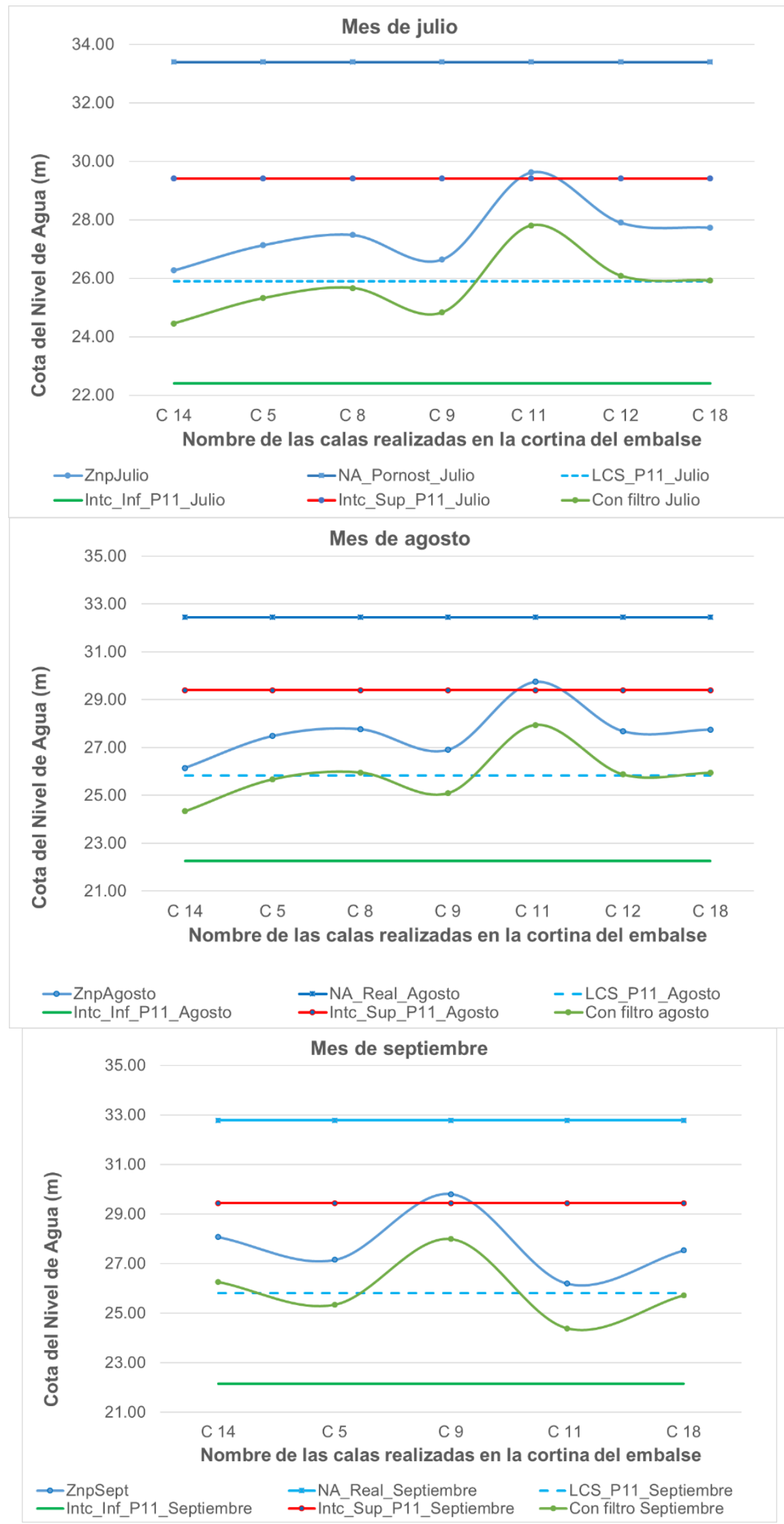

Figura 9. Evaluación de la efectividad del filtro con los resultados previos. 
El posible comportamiento ideal (LCS_P11(mes)) desde un enfoque teórico de la LCS ante un correcto proceso de drenaje del agua contenida en la estructura, se logra con la ubicación de los filtros un valor promedio del comportamiento esperado al modelo conceptual hipotético del funcionamiento de la obra. Este principio se cumple de forma efectiva para las tres geometrías evaluadas.

En consecuencia, también se decidió realizar un recálculo de los resultados del modelo Estabilidad de Taludes (TAL) donde fueron chequeados los coeficientes de seguridad y estabilidad de los taludes en las secciones objeto de estudio, principalmente en la sección central por ser la sección que geográficamente se encuentra frente a la hidroeléctrica, que es donde comienzan a surgir las filtraciones. Se toma como proceso conceptual el mostrado en la Figura 10 como resultante del análisis para la cota donde aparecen las filtraciones.



Figura 10. Comportamiento de la estabilidad del talud en el modelo TAL para la cota donde aparecen las patologías de filtraciones (37.56 m).

Las condiciones de estabilidad de taludes para los diferentes escenarios que se consideran, se comportaron de la siguiente manera: se asume un Límite Mínimo de Factor de Seguridad del Método de Bishop: 1.2, que es superado en más de 0.2 unidades en el caso más desfavorable (Método de Jambu) por lo que se identifica en todo momento una mejora a la estabilidad del talud tal como se observa en la Tabla 2.

Tabla 2. Relación de los Factores de Seguridad calculados.

\begin{tabular}{|c|r|r|r|r|r|r|r|}
\hline \multirow{2}{*}{$\begin{array}{c}\text { Método de } \\
\text { cálculo del } \\
\text { FS }\end{array}$} & \multicolumn{6}{|c|}{ Factor de Seguridad } \\
\cline { 2 - 8 } & $\begin{array}{c}\text { Antes del } \\
\text { filtro }\end{array}$ & Trapecio & Diferencia & Triángulo & Diferencia & Círculo & Diferencia \\
\hline Bishop & 1.609 & 1.606 & -0.003 & 1.606 & -0.003 & 1.606 & -0.003 \\
\hline Jambu & 1.462 & 1.495 & 0.033 & 1.495 & 0.033 & 1.495 & 0.033 \\
\hline Ordinario & 1.501 & 1.524 & 0.023 & 1.524 & 0.023 & 1.524 & 0.023 \\
\hline
\end{tabular}

\section{Conclusiones y recomendaciones}

Acorde con los resultados alcanzados, se arribaron a las siguientes conclusiones:

1. Al comparar los resultados teóricos obtenidos en función del comportamiento de los niveles de surgencia de la LCS teórica después de la colocación del filtro, en contraste con los estudios previos realizados, se obtuvieron resultados superiores a $-1.00 \mathrm{~m}$ de disminución del nivel freático teórico registrado en las diferentes calas de control y piezómetros.

2. En las modelaciones de los diferentes filtros, se alcanza una reducción del caudal que pasa por la estructura de la cortina y la base de cimentación. Estos datos fueron comparados con los medidos delante del filtro y los valores teóricos alcanzados en los modelos virtuales después de la colocación de las variantes de solución. Los resultados obtenidos fueron múltiples valores de gastos con una diferencia no mayor de $2.5 \mathrm{E}-06 \mathrm{~m} 3 / \mathrm{s}$ como evidencia en el proceso de redireccionamiento del flujo en el interior 
de la cortina por el correcto funcionamiento de la propuesta de los nuevos filtros expresado en términos de amenaza, vulnerabilidad y riesgo.

3. Luego de realizar el análisis de la estabilidad de taludes para los diferentes escenarios, se obtienen resultados que superan el valor normativo como criterio mínimo para el Factor de Seguridad del talud, por lo que se ratifica como válida la solución ingenieril empleada en el talud aguas abajo de la cortina en el embalse Zaza. Se recomienda la construcción del filtro de tipo prisma por ser muy fácil su ejecución constructiva por las empresas constructoras especializadas en trabajos hidráulicos a nivel nacional.

\section{Bibliografía}

Álvarez, L. (1998). "La estabilidad de cortinas de presas de tierra mediante la solución de los estados tenso-deformacionales y la aplicación de la teoría de seguridad". (Tesis presentada en opción al Grado Científico de Doctor en Ciencias Técnicas), Universidad Central "Marta Abreu" de Las Villas, Santa Clara, Villa Clara, Cuba.

Álvarez, M., Álvarez, L., y Martínez, J. E. (2015a). Simulación de patologías a obras hidráulicas, caso de estudio presa Zaza. La Habana, Cuba: Palacio de las Convenciones, GEOCIENCIAS' 2015.2 Recuperado de: www.redciencia.cu/geobiblio/paper/2015b_Alvarez\%20

Gonzalez.pdf(accedido el 4/09/2017).

Álvarez, M., Álvarez, L., y Martínez, J. E. (2015b). Simulación físico-numérica para evaluar el comportamiento sismo resistente en presas de tierra. Palacio de las Convenciones, La Habana, Cuba: VI Convención Cubana de Ciencias de la Tierra GEOCIENCIAS'2015, Evento: Sismología y Riesgos asociados. Recuperado de: www.redciencia.cu/geobiblio/paper/2015c_Alvarez\%20 Gonzalez.pdf(accedido el4/09/2017).

Álvarez, M., Álvarez, L., y Vázquez, R. (2017). "Visualización científica de datos geodésicos en presas de tierra, caso Embalse Palmarito". En: Ingeniería Hidráulica y Ambiental, 38(1), p. 86-100.

Armas, R. (1990). Guidelines for the design of earth dams: priority and sequence. Ciudad de La Habana, Cuba: Civil Engineering Faculty, Instituto Superior Politécnico“ José Antonio Echevarría" (ISPJAE).

Armas, R. (2002). "Criterios para diseñar presas de tierra: prioridad y secuencia". Taller Nacional sobre fallos de presas de tierra. Ciudad de La Habana, Cuba: Instituto Nacional de Recursos Hidráulicos.

Armas, R., Echemendía, A., y Garcías, C. (1994). "Causas de fallas por sifonamiento de la presa Las Cabreras": Caso histórico. Ciudad de La Habana, Cuba: Facultad de Ingeniería Civil del Instituto Superior Politécnico“José Antonio Echevarría" (ISPJAE).

Armas, R., y Horta, E. (1987). Presas de tierra. Ciudad de la Habana, Cuba: Facultad de Ingeniería Civil del Instituto Superior Politécnico "José Antonio Echevarría"(ISPJAE), Editorial ISPJAE.

Aydemir, A. y Güven, A. (2017). Modified risk assessment tool for embankment dams: case study of three dams in Turkey. En:Civil Engineering and Environmental Systems, 34(1), 53-67. DOI: 10.1080/10286608.2017.1300794

Bitner, A. (2016). Cartographic Analyzes in GIScience. Paper presented at the 2016 Baltic Geodetic Congress (BGC Geomatics).

Botello, S. (2006). Ejemplos de Aplicación de los Métodos Numéricos a Problemas de Ingeniería. Guanajuato, México: Centro de Investigación en Matemáticas A.C. (CIMAT).

Cerón López, G. M. (2017). Modelamiento geoestadístico aplicado a la caracterización de yacimientos: modelo basado en Celdas de Alta Resolución. En: Enfoque UTE, 8(4), 41. 
Daniszewska, E. (2017). Application of The Fuzzy Set Theory to Determine The Partial Factor of Safety. Paper presented at the 2017 Baltic Geodetic Congress (BGC Geomatics).

Kasireddy, V., Ergan, S., Akinci, B. y Gulgec, N. S. (2015). "Visualization requirements of engineers for risk assessment of embankment dams". En:Visualization in Engineering, 3(1), p.1. DOI: 10.1186/s40327-014-0014-y

Li, Y. \& Wang, Y. (2017). Design and implementation of reservoir dam safety monitoring platform based on ASP.NET. Paper presented at the 2017 IEEE 2nd Advanced Information Technology, Electronic and Automation Control Conference (IAEAC).

MICONS. (2005a). Manual Práctico de Hidráulica Tomo I. Ciudad de La Habana, Cuba: Instituto de Hidroeconomía. Ministerio de la Construcción (MICONS).

MICONS. (2005b). Manual Práctico de Hidráulica Tomo II. Ciudad de La Habana, Cuba: Instituto de Hidroeconomía. Ministerio de la Construcción (MICONS).

Popnicolov, A. I. (1974). Proyectos y ejercicios prácticos de complejo hidráulicos (Primera Ed., Segunda Reimpresión, 1982 ed.). Ciudad de La Habana, Cuba: Editorial Pueblo y Educación.

Sherald, J., y Woodward, R. (1963). Earth and earth-rock dams. Ciudad de La Habana, Cuba: Edición Revolucionaria, Instituto del Libro.

Vázquez, R., Pérez, C, Torres, J.C. (2015)."Exploratory data analysis through the integration of visualization techniques in geographical information systems", En: Revista Técnica de la Facultad de Ingeniería de la Universidad del Zulia.38(1), p. 73-82. 